\title{
B-WEAK COMPACTNESS OF WEAK DUNFORD-PETTIS OPERATORS
}

\author{
Belmesnaoui Aqzzouz and JaWAd H'Michane
}

Abstract. We characterize Banach lattices on which each weak Dunford-Pettis operators is bweakly compact and we derive some characterizations of KB-spaces.

Mathematics subject classification (2010): 46A40, 46B40, 46B42.

Keywords and phrases: b-weakly compact operator, weak Dunford-Pettis operator, Banach lattice, (b)property, order continuous norm, KB-space.

\section{REFERENCES}

[1] C. D. Aliprantis and O. Burkinshaw, Positive operators, Reprint of the 1985 original, Springer, Dordrecht, 2006.

[2] S. Alpay, B. Altin, and C. Tonyali, On property (b) of vector lattices, Positivity 7, 1-2 (2003), $135-139$.

[3] B. Altin, Some property of b-weakly compact operators, G.U. Journal of science 18, 3 (2005), 391395.

[4] B. Aqzzouz, A. Elbour, And J. Hmichane, The duality problem for the class of b-weakly compact operators, Positivity 13, 4 (2009), 683-692.

[5] B. Aqzzouz, And J. Hmichane, The b-weak compactness of order weakly compact operators, Complex Anal. Oper. Theory, DOI 10. 1007/s 11785-011-0138-1.

[6] B. Aqzzouz, A. Elbour, The b-weakly compactness of semi-compact operators, Acta Sci. Math. (Szeged) 76 (2010), 501-510.

[7] P. Meyer-Nieberg, Banach lattices, Universitext, Springer-Verlag, Berlin, 1991.

[8] W. WnuK, Remarks on J. R. Holub's paper concerning Dunford-Pettis operators, Math. Japon 38 (1993), 1077-1080. 